\title{
Proteomic analysis and comparison of intra- and extracranial cerebral atherosclerosis responses to hyperlipidemia in rabbits
}

\author{
ZHI-LAN TU ${ }^{1,2}$, BO YU ${ }^{2}$, DONG-YA HUANG ${ }^{1}$, RAJEEV OJHA ${ }^{1}$, SHU-KUI ZHOU ${ }^{1}$, HE-DI AN ${ }^{1}$, \\ RONG LIU ${ }^{1}$, CUI DU ${ }^{1}$, NAN SHEN ${ }^{1}$, JIAN-HUI FU ${ }^{2}$ and SHUANG-XING HOU ${ }^{2}$ \\ ${ }^{1}$ Department of Neurology, East Hospital, Tongji University School of Medicine, Shanghai 200120; ${ }^{2}$ Department of \\ Neurology, Shanghai Pudong Hospital, Fudan University Pudong Medical Center, Shanghai 201399, P.R. China
}

Received January 10, 2016; Accepted January 20, 2017

DOI: $10.3892 / \mathrm{mmr} .2017 .6869$

\begin{abstract}
The present study aimed to investigate protein expression levels of intra- and extracranial atherosclerosis in rabbits following administration of a high-fat diet. Rabbits were randomly divided into control (group $\mathrm{A} ; \mathrm{n}=9$ ) and high-fat diet (group B; n=9) groups. At week 12, tissues were sectioned from the common carotid artery (CCA) and middle cerebral artery (MCA). Pathological analysis was performed. Differential protein expression levels were examined by 2-D gel electrophoresis (2-DE) and mass spectrometry (MS) analysis and validated by western blotting. Serum lipid levels, the intima-media thickness (IMT) and degree of atherosclerosis of the CCA and MCA were increased at week 12 in the high-fat diet group compared with rabbits that received a normal diet. 2-DE and MS analysis of the protein extracted from CCA and MCA detected $>439$ different proteins; the expression of 25 proteins was altered, and 8 proteins [albumin A chain, tropomyosin $\alpha-1$ chain (TPM1), heat shock protein 70 (HSP70), $\alpha$-smooth muscle actin, $\beta$-galactose binding agglutinin, TPM4 isoform 2 , cell keratin 9 , single octylic acid glyceride $\beta-2$ ) demonstrated significant alterations in expression levels. Due to limited antibody sources, only three differentially expressed proteins (TPM1, HSP70 and $\alpha$-smooth muscle actin) were examined by western blotting. The results of our previous study demonstrated that hyperlipidemia affected the IMT of intracranial and extracranial cerebral arteries. In the present study, protein expression levels of TPM1 and $\alpha$-smooth muscle actin from extracranial cerebral arteries were significantly increased compared with intracranial cerebral arteries; however, protein expression levels of HSP70 from intracranial cerebral arteries was increased compared with extracranial cerebral arteries. The differences may be closely associated
\end{abstract}

Correspondence to: Professor Dong-Ya Huang, Department of Neurology, East Hospital, Tongji University School of Medicine, 150 Jimo Road, Pudong, Shanghai 200120, P.R. China

E-mail: dongyahuangcn@163.com

Key words: hyperlipidemia, intracranial and extracranial cerebral atherosclerosis, proteomic analysis, western blotting with cell proliferation and metastasis, and oxidoreduction, in intra- and extracranial cerebral atherosclerosis. HSP70 may have protective properties against atherosclerosis via underlying anti-inflammatory mechanisms, furthermore, differential protein expression levels (TPM1, HSP70 and $\alpha$-smooth muscle actin) between intra- and extracranial cerebral arteries may facilitate the identification of novel biological markers for the diagnosis and treatment of cerebral arteriosclerosis.

\section{Introduction}

Cerebral atherosclerosis, the primary cause of ischemic stroke, is divided into extracranial atherosclerosis (ECAS) and intracranial atherosclerosis (ICAS) (1-3). The underlying molecular mechanisms of ICAS and ECAS have been extensively studied; however, remain to be fully elucidated. Cerebral atherosclerosis is influenced by various risk factors, including hypertension, hyperlipidemia, obesity, smoking and diabetes. Previous studies have reported that blood cholesterol levels and associated lipoprotein levels are associated with the risk of coronary artery disease; however, this is weakly associated in ECAS and ICAS, suggesting potential variations in underlying mechanisms or susceptibility $(2,4,5)$. The role of hyperlipidemia in ICAS and ECAS remains unclear due to controversial previously reported findings. A previous study conducted proteomics analyses of the common carotid artery by obtaining human carotid atherosclerotic plaques, and demonstrated certain proteins are in low-abundance, including heat shock protein 27 (HSP27) isoforms, aldehyde dehydrogenase, moesin, protein kinase $\mathrm{C} \delta$-binding protein and inter- $\alpha$-trypsin inhibitor family heavy chain-related protein are correlated with biological alterations associated with atherosclerosis (6). In our previous study, HSP70 expression levels were demonstrated to vary between ICAS and ECAS; however, the effect of other protein expressions on ICAS and ECAS remains unclear (7). Investigation into the impact of hyperlipidemia on ICAS and ECAS have yielded controversial results, and it is unclear whether any specific target proteins are involved in the discrepancies between ICAS and ECAS. A more global analysis of the activities of all relevant molecules is required, which may provide a complete view of their interactions. Proteomic analysis has previously been performed to identify novel therapeutic targets for use in the treatment 
and prevention of atherosclerosis (8). The present study aimed to compare proteomic and biomarker profiles associated with cerebral ICAS and ECAS. The identification of protein biomarkers that underlie cerebral atherosclerosis may provide valuable diagnostic indicators and therapeutic targets for the treatment of the disease.

\section{Materials and methods}

Animals. Male New Zealand White rabbits ( $\mathrm{n}=18$; weight, 2.0-2.5 kg) were provided by the Animal Laboratory of Tongji University (Shanghai, China). They were fed regular rabbit feed for one week prior to the experiment and were kept in a temperature-controlled environment $\left(20 \pm 1^{\circ} \mathrm{C}\right.$; humidity, $55 \pm 5 \%$ ) under a 12 -h light/dark cycle in an air-conditioned room. Rabbits were randomly divided into two groups: Control $(A ; n=9)$ and high-fat $(B ; n=9)$. Rabbits in group A continued to receive regular rabbit feed and group $\mathrm{B}$ were fed a high-fat diet ( $2 \%$ cholesterol, $6 \%$ peanut oil and $92 \%$ basic feed) for 12 weeks based on the methods of previous studies $(6,9,10)$. Rabbits in groups A and B were fed restricted diets with an equal amount of food (150-200 g per day) and free access to water. The present study was carried out in strict accordance with the recommendations in the Guide for the Care and Use of Laboratory Animals of the National Institutes of Health. The animal use protocol was reviewed and approved by the Institutional Animal Care and Use Committee of Tongji University School of Medicine (Shanghai, China).

Lipid profile and histopathological examination. Circulation blood samples were collected from the rabbits at weeks 0,4 , 8 and 12 on the different diets. The rabbits in groups A and B were sacrificed at weeks $0,4,8$ and 12 . For groups A and B, 3 rabbits were sacrificed at each time point (4,8 and 12 weeks). Artery segments [including the common carotid artery (CCA) and middle cerebral artery (MCA)] were dissected and fixed in $4 \%$ paraformaldehyde for histomorphometry observation. Pathological analysis was performed by hematoxylin and eosin (H\&E) staining, as described previously (7). Images were observed under a light microscope, following which intima-media thickness (IMT) was measured using Image Pro Plus software version 6.0 (Media Cybernetics, Inc., Rockville, $\mathrm{MD}, \mathrm{USA})$. The tissues were stored at $-80^{\circ} \mathrm{C}$ for $2-\mathrm{D}$ gel electrophoresis (2-DE) and proteins expression levels were analyzed by western blotting.

2-DE and silver staining. CCA and MCA tissues from groups A and $\mathrm{B}$ were ground to a powder using liquid nitrogen, detailed process as follows: Homogenized in ice-cold homogenizing buffer [9 M urea; 4\% w/v CHAPS; $1 \% \mathrm{w} / \mathrm{v}$ dithiothreitol (DTT); $0.5 \%$ chilled acetone (CA) and a cocktail of protease inhibitors]. The homogenate was centrifuged at 3,500 x g for $30 \mathrm{~min}$ at $6^{\circ} \mathrm{C}$ and the precipitation was collected and suspended in precooling acetone containing $0.2 \%$ DTT, and frozen at $-20^{\circ} \mathrm{C}$ for $1 \mathrm{~h}$. Precipitated proteins were centrifuged for $30 \mathrm{~min}$ at $5,000 \mathrm{x} \mathrm{g}$ at $6^{\circ} \mathrm{C}$, which was followed by two additions of CA. The pellets were air dried at room temperature and dissolved overnight at $4^{\circ} \mathrm{C}$ in lysis buffer containing $7 \mathrm{M}$ urea, $2 \mathrm{M}$ thiourea, $4 \% \mathrm{w} / \mathrm{v}$ CHAPS, $1 \%$ w/v DTT, $0.5 \%$ ampholyte and a cocktail of protease inhibitors. Tissue proteins were fractionated as described previously (11-13) and protein concentrations were determined using the Bradford assay according to methods described previously (14), and subsequently stored at $-80^{\circ} \mathrm{C}$ for isoelectric focusing (IEF).

2-DE and silver staining were performed. Protein sample $(200 \mu \mathrm{g})$ was mixed with fresh rehydration buffer to a total of $450 \mu 1$, following which IEF was performed using an Immobiline DryStrip (IDS) gel (GE Healthcare Life Sciences, Chalfont, UK; length, $24 \mathrm{~cm}$; $\mathrm{pH} 3-10$; non-linear gradient) at room temperature for $10 \mathrm{~min}$. Following a two-step equilibration, proteins on the IPG strips were separated by SDS-PAGE using the Ettan-DALTsix Electrophoresis system (GE Healthcare Life Sciences) at $15^{\circ} \mathrm{C}$ and $200 \mathrm{~V}$ for $6-8 \mathrm{~h}$. The IDS gel was visualized by silver staining according to the protocol of Shevchenko et al (15). The stained gel was scanned using an ImageScanner system (GE Healthcare Bio-Sciences, Pittsburgh, PA, USA) at a resolution of 300 dots per inch. All gel images were processed by three steps using PDQuest 2-D Analysis software version 8.0 (Bio-Rad Laboratories, Inc., Hercules, CA, USA) as follows: Spot detection, volumetric quantification and matching. Differences in protein content between high-fat diet and control groups were analyzed by Student's t-test $(\mathrm{n}=3)$ and calculated as fold ratio. Fold-change $\geq 1.5$ or $\leq 0.67$ was used to select differentially expressed protein spots.

Mass spectrometry (MS) analysis. The extracted protein samples were re-suspended with $5 \mu 10.1 \%$ trifluoroacetic acid (TFA) followed by mixing in a matrix consisting of a saturated solution of $\alpha$-cyano-4-hydroxy-trans-cinnamic acid in $50 \%$ acetonitrile and $0.1 \%$ TFA at a $1: 1$ ratio. Mixture (1 $\mu \mathrm{l})$ was spotted onto a stainless-steel sample target plate. Peptide MS and MS/MS were performed on an AB SCIEX 5800 time-of-flight (TOF)/TOF ${ }^{\mathrm{TM}}$ system mass spectrometer (Applied Biosystems; Thermo Fisher Scientific, Inc., Waltham, MA, USA). MS and MS/MS datasets were integrated and processed using GPS Explorer ${ }^{\mathrm{TM}}$ software version 3.6 (Applied Biosystems; Thermo Fisher Scientific, Inc.) with default parameters. Proteins were successfully identified based on $95 \%$ or higher confidence interval of their scores in the Mascot v2.1 search engine (Matrix Science, Ltd., London, UK) using the Mascot and NCBI protein databases. Peptide mixtures were separated on an UltiMate ${ }^{\mathrm{TM}} 3000$ Nano Liquid Chromatography system (Dionex; Thermo Fisher Scientific, Inc.) as described by Moller et al (11).

Western blotting. Proteins were extracted as described above and protein lysates (20 $\mu \mathrm{g}$ per lane) were separated by one-dimensional $10 \%$ SDS-PAGE and were transferred onto nitrocellulose membranes. Membranes were blocked with PBS containing 5\% nonfat dry milk at $4^{\circ} \mathrm{C}$ overnight and were subsequently incubated with the following primary antibodies: Polyclonal mouse anti- $\alpha$-smooth actin (cat. no. ab7817, 1:300), mouse anti-HSP70 (cat. no. ab2787, 1:100), rabbit anti-tropomyosin $\alpha-1$ chain (cat. no. ab55915, TPM1; 1:500) (all from Abcam, Cambridge, UK) and mouse GAPDH (cat. no. sc-365062; 1:1,000; Santa Cruz Biotechnology, Inc., Dallas, TX, USA) overnight at $4^{\circ} \mathrm{C}$. The membranes were washed three times and incubated with secondary antibody horseradish peroxidase (HRP)-conjugated goat anti-rabbit 
Table I. Blood lipids levels in group A and B rabbits.

\begin{tabular}{lcccc}
\hline Treatment group & HDL $(\mathrm{mmol} / \mathrm{l})$ & LDL $(\mathrm{mmol} / \mathrm{l})$ & TG $(\mathrm{mmol} / \mathrm{l})$ & $\mathrm{CHOL}(\mathrm{mmol} / \mathrm{l})$ \\
\hline Group A & & & & \\
Week 0 & $0.42 \pm 0.07$ & $0.31 \pm 0.05$ & $0.78 \pm 0.09$ & $0.97 \pm 0.07$ \\
Week 4 & $0.56 \pm 0.15$ & $0.40 \pm 0.08$ & $0.59 \pm 0.24$ & $1.15 \pm 0.14$ \\
Week 8 & $0.49 \pm 0.08$ & $0.37 \pm 0.18$ & $1.54 \pm 0.10$ & $0.81 \pm 0.03$ \\
Week 12 & $0.48 \pm 0.03$ & $0.45 \pm 0.12$ & & $1.27 \pm 0.01$ \\
Group B & & & $0.76 \pm 0.08$ & $0.59 \pm 0.08$ \\
Week 0 & $0.41 \pm 0.04$ & $0.32 \pm 0.02$ & $33.48 \pm 8.07^{\mathrm{a}}$ \\
Week 4 & $6.65 \pm 0.91^{\mathrm{a}}$ & $31.10 \pm 12.47^{\mathrm{a}}$ & $0.79 \pm 0.36$ & $4.69 \pm 1.37$ \\
Week 8 & $9.00 \pm 3.00^{\mathrm{a}}$ & $47.96 \pm 10.28^{\mathrm{a}}$ & $4.22 \pm 2.88^{\mathrm{a}}$ & $41.27 \pm 0.93^{\mathrm{a}}$ \\
Week 12 & $10.93 \pm 0.13^{\mathrm{a}}$ & $50.80 \pm 2.25^{\mathrm{a}}$ & & $4.96^{\mathrm{a}}$ \\
\hline
\end{tabular}

${ }^{\mathrm{a}} \mathrm{P}<0.05$ vs. group A at equivalent week. Data are expressed as the mean \pm standard deviation $(\mathrm{n}=3)$. HDL, high density lipoprotein; LDL, low density lipoprotein; TG, triglyceride; CHOL, cholesterol.

IgG (cat. no. ab97051; 1:5,000) and goat anti-mouse IgG (cat. no. ab97023; 1:10,000) (both from Abcam) for $2 \mathrm{~h}$ at room temperature. Membranes were washed four times with TBST for $40 \mathrm{~min}$. Proteins were visualized using Enhanced Chemiluminescence reagents (GE Healthcare Life Sciences) and densitometry was performed using Quantity One software 4.2 (Bio-Rad Laboratories, Inc.).

Statistical analysis. Differences between intra- and extracranial cerebral atherosclerosis were evaluated by Student's t-test for normally distributed data, or the Mann-Whitney rank sum test for nonparametric data. Data are expressed as the mean \pm standard deviation. $\mathrm{P}<0.05$ was considered to indicate a statistically significant difference.

\section{Results}

Lipid profile. Group B rabbits were fed an atherogenic diet. Mean cholesterol (CHOL), low density lipoprotein (LDL), high density lipoprotein (HDL) and triglyceride (TG) levels $(\mathrm{mmol} / \mathrm{l})$ were increased in group B compared with group $\mathrm{A}$ at the end of week 12 as follows: CHOL increased from $1.27 \pm 0.01$ (Group A) to $41.27 \pm 0.93$ (Group B), LDL from $0.45 \pm 0.12$ to $50.80 \pm 2.25$, HDL from $0.48 \pm 0.03$ to $10.93 \pm 0.13$, and TG from $1.54 \pm 0.10$ to $4.22 \pm 2.88$. Various lipid levels in different groups were revealed to increase over time in group B; in particular, LDL and CHOL levels were markedly increased. In group A, blood lipids remained within the healthy range. A statistically significant difference was observed in lipid levels between the two groups $(\mathrm{P}<0.05$; Table I). These results suggested that an atherogenic diet resulted in hyperlipidemia.

Pathological sections. Pathological HE-stained sections of the CCA in groups A (Fig. 1A; magnification, x4) and B (Fig. 1B; magnification, $\mathrm{x} 4$ ), and of the MCA in groups A (Fig. 1C; magnification, $\mathrm{x} 20$ ) and $\mathrm{B}$ (Fig. D; magnification, $\mathrm{x} 4$ ) revealed that the degree of AS lesions was increased in the CCA compared with the MCA between different groups at week 12 .
Table II. Comparison of cerebral artery diameters in different groups.

\begin{tabular}{lcc}
\hline $\begin{array}{l}\text { Treatment } \\
\text { group }\end{array}$ & $\begin{array}{c}\text { Common carotid } \\
\text { artery diameter }(\mu \mathrm{m})\end{array}$ & $\begin{array}{c}\text { Middle cerebral artery } \\
\text { diameter }(\mu \mathrm{m})\end{array}$ \\
\hline $\begin{array}{l}\text { Group A } \\
\text { Week 4 }\end{array}$ & $123.0 \pm 5.77$ & $31.6 \pm 1.3$ \\
Week 8 & $129.3 \pm 13.4$ & $36.1 \pm 2.6$ \\
Week 12 & $150.2 \pm 0.5$ & $39.4 \pm 4.9$ \\
Group B & & $58.9 \pm 2.04^{\mathrm{a}}$ \\
Week 4 & $166.5 \pm 5.97^{\mathrm{a}}$ & $72.2 \pm 2.9^{\mathrm{a}}$ \\
Week 8 & $176.4 \pm 6.6^{\mathrm{a}}$ & $81.1 \pm 2.3^{\mathrm{a}}$ \\
Week 12 & $199.9 \pm 18.6^{\mathrm{a}}$ & \\
\hline
\end{tabular}

${ }^{a} \mathrm{P}<0.05$ vs. group A at equivalent week. Data are expressed as the mean \pm standard deviation $(n=3)$.

Therefore, a simple high-fat diet may lead to the formation of AS further aggravate the degree of AS lesions.

Comparison of intra- and extracranial cerebral artery segments. Previous reports have indicated that IMT measurements are a representative end point for AS and vascular disease risk (16). IMT measurements provide data on the efficacy of novel lipid-modifying techniques following a high-fat diet. To estimate AS progression between groups A and B, cross-sectional standardized IMT measurements were used, and because measurements were standardized, AS progression estimates were extrapolated from the cross-sectional data for each group. The IMT of the CCA and MCA were measured in all animals and combined to a per-subject average. IMT of the CCA and MCA increased over the 12 weeks in both groups; however, after 4,8 and 12 weeks of receiving the different diets the CCA and MCA IMT was significantly increased in group $\mathrm{B}$ compared with group A $(\mathrm{P}<0.05$; Table II). Thus, a high-fat diet can cause IMT thickness in different cerebral arteries. 

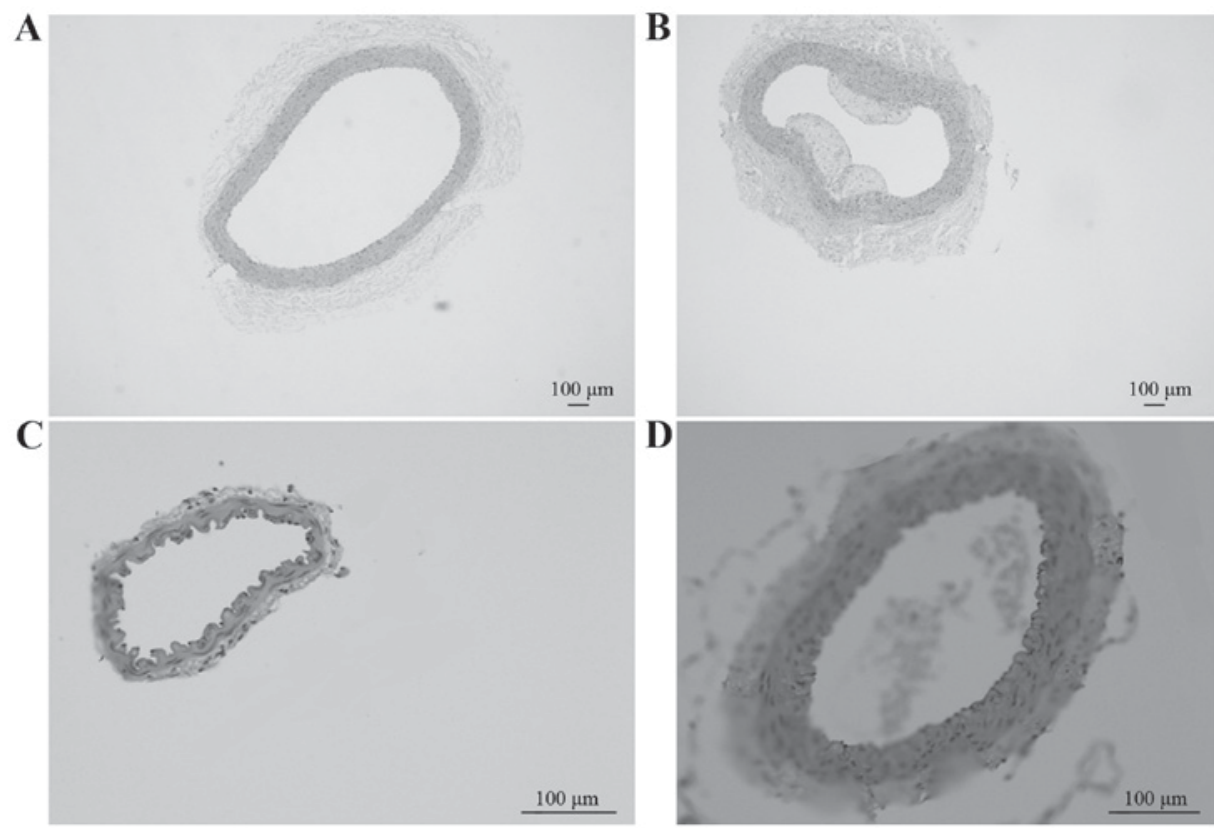

Figure 1. Hematoxylin-eosin-stained sections of arteries at week 12. Common carotid artery sections from (A) group A demonstrated smooth endomembrane and (B) in group B fatty streak formation plaque could be observed (magnification, $x 4$ ). Middle cerebral artery sections from (C) group A had smooth endomembrane and (D) in group B the inhomogeneous intima-media was thickened (magnification, x20). Scale bar, $100 \mu \mathrm{m}$. Group A, control group; group B, high-fat diet group.
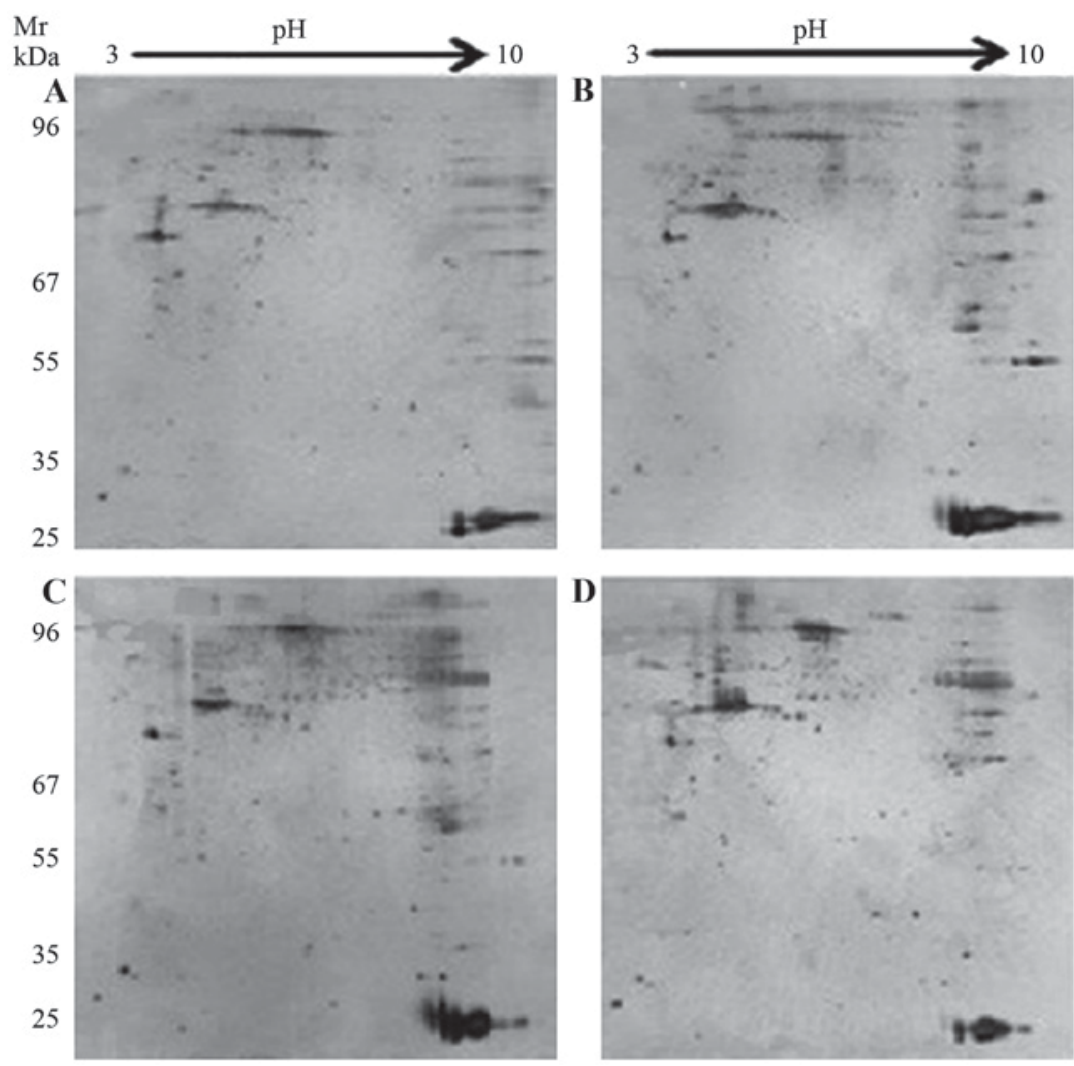

Figure 2. A representative 2-D gel map of CCA and MCA sections from groups A and B. Total protein extracts from (A) CCA in group A, (B) CCA in group $\mathrm{B}$, (C) MCA in group A and (D) MCA in group B were separated on $\mathrm{pH}$ 3-10 nonlinear immobilized $\mathrm{pH}$ gradient strips in the first dimension followed by SDS-PAGE in the second dimension. The gels were visualized. In total, 8 altered spots were identified by 2-D gel electrophoresis and mass spectrometry analyses. Data on each of these protein species is reported in Table III. CCA, common carotid artery; MCA, middle cerebral artery; group A, control group; group B, high-fat diet group.

Proteomic analysis. To investigate the key proteins associated with ICAS and ECAS, 2-DE and silver staining were performed. 2-D gel images of proteins isolated from the MCA and CCA of groups A and B are presented in Fig. 2. Total 
Table III. Differentially expressed proteins in intra- and extracranial cerebral arteries.

\begin{tabular}{|c|c|c|c|c|}
\hline Spot no. ${ }^{\text {a }}$ & Accession no. & Protein ID $^{\mathrm{b}}$ & $\mathrm{kDa}$ & Mascot score \\
\hline 3203 & gi126723746 & Precursor protein & 70.861 & 60 \\
\hline 2902 & gi148594078 & Heat shock protein 70 & 71.424 & 105 \\
\hline 4201 & gil49864 & $\alpha$-smooth muscle actin & 38.016 & 107 \\
\hline 5001 & gil291414651 & $\beta$-galactose binding agglutinin & 15.170 & 118 \\
\hline 202 & gil4507651 & Tropomyosin $\alpha-4$ chain isoform 2 & 28.619 & 91 \\
\hline 2901 & gil435476 & Cell keratin 9 & 62.320 & 113 \\
\hline 1203 & gi112448 & Tropomyosin $\alpha-1$ chain & 32.233 & 49 \\
\hline 8201 & gil395805236 & Singleoctylic acid glyceride $\beta 2$ & 69.636 & 53 \\
\hline
\end{tabular}

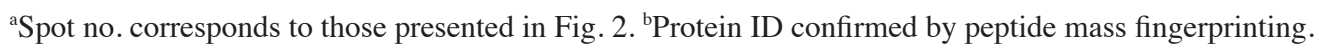
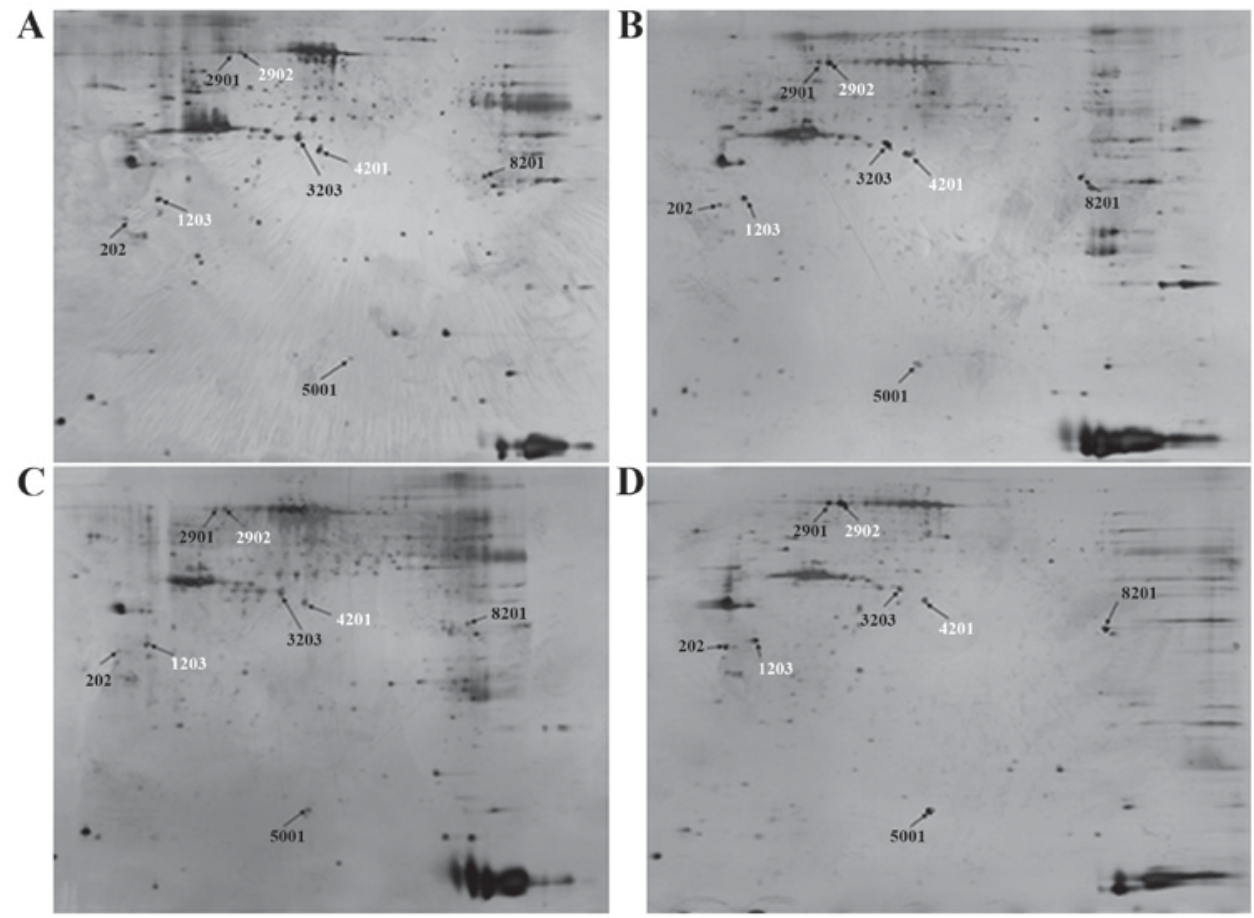

Figure 3. Protein expression differences in groups A and B, as analyzed by mass spectrometry. (A) CCA in group A, (B) CCA in group B, (C) MCA in group A and (D) MCA in group B. Black arrows indicate proteins that were significantly altered following a high-fat diet; $>439$ protein spots were detected per gel. Of the altered proteins, 25 protein expression levels were different and 8 spots (marked with arrow and number) demonstrated significant alterations and were quantitatively high enough to be identified by Matrix-assisted laser desorption/ionization-time-of-flight tandem mass spectrometry. CCA, common carotid artery; MCA, middle cerebral artery; group A, control group; group B, high-fat diet group.

protein extracts from different groups were separated on 1-D nonlinear IPG strips ( $\mathrm{pH} 3-10)$ followed by 2-D SDS-PAGE. Spectrum analysis was used to identify protein spots, with $>2$-fold or $<0.5$-fold-changes as a standard. Per gel, $>439$ protein spots were detected, and well-matched spots and control gels were analyzed for comparative proteomics. Of the altered proteins, 25 protein expression levels had significant alterations, and were quantitatively increased enough to be identified by matrix-assisted laser desorption/ionization (MALDI)-TOF/TOF. MS data were analyzed using the Mascot search engine. All 25 spots were identified with high confidence by comparing $>70 \%$ different degree groups. The present study identified that protein expression levels of 25 proteins were significantly increased in the MCA and CCA of group B compared with group A using Mascot retrieval software. A total of 8 differential proteins were observed: Precursor protein (albumin A chain), TPM1, HSP70, $\alpha$-smooth muscle actin, $\beta$-galactose binding agglutinin, TMP4 isoform 2 , cell keratin 9 and single octylic acid glyceride- $\beta$ (Fig. 3; Table III).

Western blot analysis. Based on the fold-change results and the protein biological functions, the following proteins were selected for further evaluation by western blotting: TPM1, HSP70 and $\alpha$-smooth muscle actin. GAPDH served as an internal control. Western blot images of protein expression levels of TPM1, $\alpha$-smooth muscle actin and HSP70 from MCA and CCA in groups A and B are presented in Fig. 4A. Protein expression was semi-quantitatively evaluated using 
A

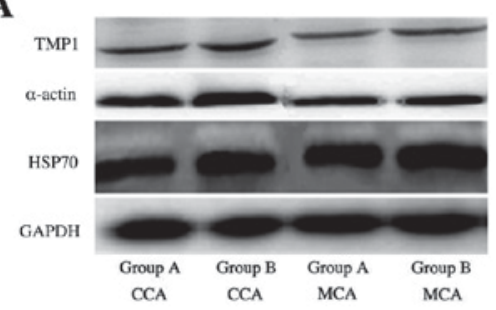

B

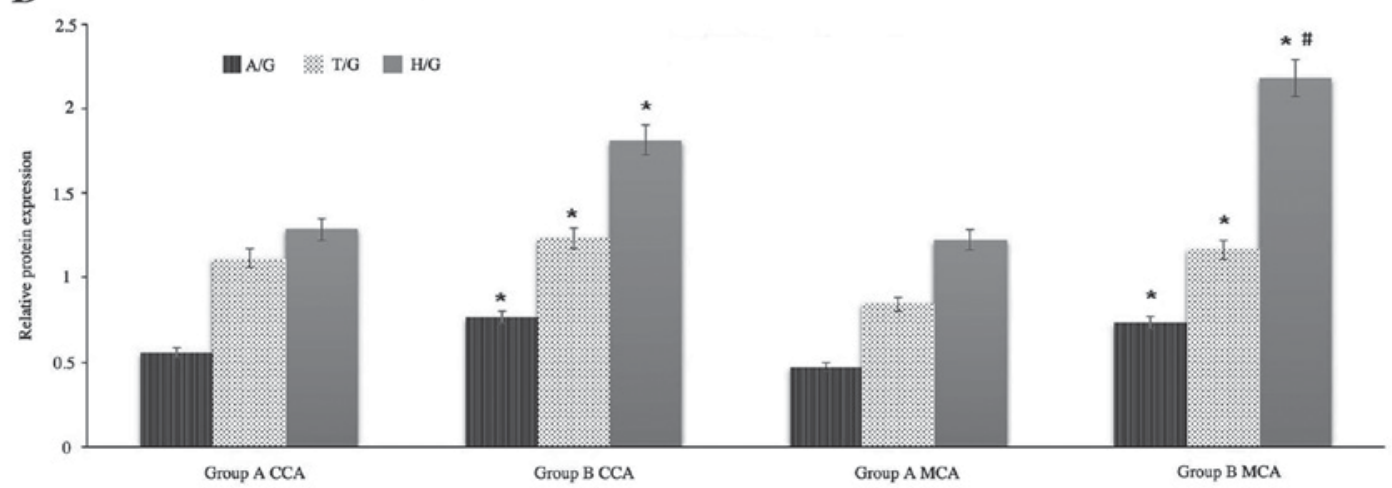

Figure 4. Western blot analysis. (A) Representative western blot images and (B) quantification of TPM1, $\alpha$-smooth muscle actin and HSP70 protein expression levels in the CCA and MCA of groups A and B. GAPDH served as an internal control. Data are expressed as the mean \pm standard deviation from two experiments. " $\mathrm{P}<0.05$ vs. group A CCA and ${ }^{\#} \mathrm{P}<0.05$ vs. group B CCA. TPM1, tropomyosin $\alpha-1$ chain; HSP70, heat shock protein $70 ; \alpha$-actin, $\alpha$-smooth muscle actin; CCA, common carotid artery; MCA, middle cerebral artery; group A, control group; group B, high-fat diet group; A/G, $\alpha$-smooth muscle actin/GAPDH; T/G, TPM1/GAPDH; H/G, HSP70/GAPDH.

densitometry (Fig. 4B). The results revealed that the expression of $\alpha$-smooth muscle actin and TPM1 chain from CCA in group $\mathrm{B}$ are increased marginally compared with from MCA in group B, however, the expression of HSP70 from MCA in group B is significantly increased compared with from CCA in group B. It was consistent with the results of mass spectrum identification.

\section{Discussion}

Biomarkers including C-reactive protein, B-typenatriuretic peptides and cardiac troponins have previously been used by clinicians as predictors of future cardiovascular events $(17,18)$. However, highly reliable biomarkers of cerebrovascular events remain to be identified. Existing proteomic studies on nervous system disease have primarily focused on penumbral phenomena, movement disorders and neurodegenerative disease (19-23). In the present study, a proteomics approach was used to identify if protein expression levels were altered in response to ICAS and ECAS based on the controversy between hyperlipidemia and cerebral atherosclerosis. Previous studies have investigated the differences in risk factors and stroke mechanisms between ICAS and ECAS, and demonstrated that hyperlipidemia is more closely associated with ECAS (2), whereas hyperlipidemia was more closely associated with MCA stenosis (5). The differences of protein expressions in high-fat-fed rabbits between ICAS and ECAS are still unclear. In order to provide information about the possible mechanisms of atherosclerosis progression and identify potential therapeutic target molecules for ICAS and ECAS, a global proteomic analysis of different cerebral arteries was performed in the present study.
The present study demonstrated that mean CHOL, LDL, HDL and TG levels significantly increased by week 12 following a high-fat diet compared with rabbits that had received a normal diet. Lipid levels were increased over time in group B compared with group A; in particular, LDL and CHOL levels were markedly increased. It has previously been reported that IMT measurements are a representative end point for AS and vascular disease (16). To estimate AS progression following a high-fat diet, IMT of the CCA and MCA were measured in all rabbits. At week 12, the IMT and AS degree of CCAs and MCAs was significantly increased in group B compared with group A. These data indicated the successful establishment of a cerebral AS model of rabbits with a high-fat diet, and that hyperlipidemia is positively associated with intra- and extracranial cerebral AS. Proteomic analysis and comparison of intra- and extracranial cerebral AS responses to hyperlipidemia in rabbit was performed by 2-DE and MS analyses. The present study identified an average of 439 different proteins in the analyzed samples; 25 proteins were differentially expressed in group A compared with group B, and 8 spots were significantly different; these 8 spots were quantitatively increased enough to be identified by MALDI-TOF/TOF analysis. Only three of the significantly expressed proteins (TPM1, HSP70 and $\alpha$-smooth muscle actin) were examined by western blot analysis due to limited antibody sources.

$\alpha$-smooth muscle actin is a component of the contractile apparatus in muscle cells, and is an important marker of AS plaque formation. Loss of $\alpha$-smooth muscle actin reduces the contractile ability of the cell, increases smooth muscle actin proliferation and leads to excessive neointimal formation with vascular injury $(24,25)$. The majority of upregulated 
$\alpha$-smooth muscle actin expression may be involved in early remodeling that occurs prior to marked adaptive alterations (26). The present study demonstrated the upregulation of $\alpha$-smooth muscle actin expression in ICAS and ECAS in rabbits that received a high-fat diet compared with rabbits on normal diet, and the expression of $\alpha$-smooth muscle actin from extracranial cerebral arteries was significantly increased compared with intracranial cerebral arteries. Increased expression levels of $\alpha$-smooth muscle actin following a high-fat diet may enhance inflammation of injured vessels, leading to proliferation of vessel smooth muscle actin. The increase of vessel smooth muscle actin and intimal hyperplasia of arteries is the primary pathological event leading to stenosis.

Simoneau et al (27) demonstrated that TPM1 may regulate endothelial barrier integrity in response to oxidative stress conditions, and phosphorylation of TPM1 at Ser283 regulates endothelial permeability and transendothelial migration of cancer cells. The primary finding of the present study was that TPM1 expression levels were abundantly increased in CCAs and MCAs by a high-fat diet. TPM1 expression was semi-quantitatively evaluated using densitometry, and the results demonstrated that the expression of TPM1 in CCA was increased marginally compared with MCA in group $\mathrm{B}$; the average intensity of TPM1/GAPDH in CCA was 1.232 and the average intensity of TPM1/GAPDH in MCA was 1.164, which indicates that extracranial cerebral arteries are more susceptible to endothelial damage in AS than intracranial cerebral arteries.

HSP70 has been demonstrated to suppress inflammation and tissue damage via an underlying mechanism that involves an enhanced regulatory response mediated by antigen-specific interleukin-10 production (28). Overexpression of HSP70 serves an important role in cell death and has neuroprotective effects via an anti-inflammatory underlying mechanism, and has been demonstrated to be positively associated with ICAS and ECAS $(29,30)$. However, whether ICAS or ECAS is more closely associated with altered expression levels of HSP70 remains to be elucidated. Furthermore, expression levels of HSP70 from intracranial cerebral arteries were significantly increased compared with extracranial cerebral arteries in this study, in contrast to the expression patterns of TPM1 and $\alpha$-smooth muscle actin. Therefore, HSP70 may have AS-protective properties via anti-inflammatory underlying mechanisms in ICAS compared with ECAS. This finding is consistent with a previous study, which examined the proteomic analysis of stable and unstable carotid atherosclerotic plaques (6). The findings of the present study may partly explain previous contradictory observations on the differences in cerebral AS mechanisms.

The present study demonstrated that hyperlipidemia exerts varying influences on intra- and extracranial cerebral arteries. Proteomic analysis revealed that 8 proteins exhibited varying degrees of differential expression in intra- and extracranial cerebral arteries, which are involved in oxidative stress, tumor metastasis, inflammation, cholesterol transport, cell apoptosis and adhesion. Alterations in protein expression levels of $\alpha$-smooth muscle actin, TPM1 and HSP70 may be involved in the differences between ICAS and ECAS. The underlying mechanisms of these proteins on the formation of AS require clarification. Further investigating the pathogenesis of differential protein expression levels between intra- and extracranial cerebral arteries may facilitate the identification of novel biological markers for the diagnosis and treatment of cerebral arteriosclerosis.

\section{Acknowledgements}

The present study was supported by the Science and Technology Foundation of Shanghai (grant no. 13JC1407103 2013-2016), Foundations for the Young Academic Leader of Tongji University (grant no. 1500144001), the Health Science and Technology of Pudong Municipal Commission of Health and Family Planning of Shanghai (grant no. PW2013D-13) and Puxiu Plan of Shanghai Pudong Hospital (grant no. PX201501).

\section{References}

1. Kim J, Cha MJ, Lee DH, Lee HS, Nam CM, Nam HS, Kim YD and $\mathrm{Heo} \mathrm{JH}$ : The association between cerebral atherosclerosis and arterial stiffness in acute ischemic stroke. Atherosclerosis 219: 887-891, 2011.

2. Kim JS, Nah HW, Park SM, Kim SK, Cho KH, Lee J, Lee YS, Kim J, Ha SW, Kim EG, et al: Risk factors and stroke mechanisms in atherosclerotic stroke: Intracranial compared with extracranial and anterior compared with posterior circulation disease. Stroke 43: 3313-3318, 2012.

3. Soloperto G and Casciaro S: Progress in atherosclerotic plaque imaging. World J Radiol 4: 353-371, 2012.

4. Prabhakaran S and Romano JG: Current diagnosis and management of symptomatic intracranial atherosclerotic disease. Curr Opin Neurol 25: 18-26, 2012.

5. Wong KS, Ng PW, Tang A, Liu R, Yeung V and Tomlinson B: Prevalence of asymptomatic intracranial atherosclerosis in high-risk patients. Neurology 68: 2035-2038, 2007.

6. Malaud E, Piquer D, Merle D, Molina L, Guerrier L, Boschetti E, Saussine M, Marty-Ané C, Albat B and Fareh J: Carotid atherosclerotic plaques: Proteomics study after a low-abundance protein enrichment step. Electrophoresis 33: 470-482, 2012.

7. Tu Z, Huang D, Yang J, Ojha R, Xiao Y, Liu R, Du C, Shen N, An H, Yu F, et al: Effect of dyslipidemia on intima-media thickness of intra- and extracranial atherosclerosis by regulating the expression of hsp70 in rabbits. Int J Clin Exp Med 8: 5446-5453, 2015.

8. Barderas MG, Vivanco F and Alvarez-Llamas G: Vascular proteomics. Methods Mol Biol 1000: 1-20, 2013.

9. Dol F, Schaeffer P, Lamarche I, Mares AM, Chatelain P and Herbert JM: Effect of SR 33805 on arterial smooth muscle cell proliferation and neointima formation following vascular injury. Eur J Pharmacol 280: 135-142, 1995.

10. Richardson M, Hatton MW, Buchanan MR and Moore S: Wound healing in the media of the normolipemic rabbit carotid artery injured by air drying or by balloon catheter de-endothelialization. Am J Pathol 137: 1453-1465, 1990.

11. Moller MJ, Qin Z and Toursarkissian B: Tissue markers in human atherosclerotic carotid artery plaque. Ann Vasc Surg 26: 1160-1165, 2012.

12. Piubelli C, Carboni L, Becchi S, Mathé AA and Domenici E: Regulation of cytoskeleton machinery, neurogenesis and energy metabolism pathways in a rat gene-environment model of depression revealed by proteomic analysis. Neuroscience 176: 349-380, 2011.

13. Tannu NS, Howell LL and Hemby SE: Integrative proteomic analysis of the nucleus accumbens in rhesus monkeys following cocaine self-administration. Mol Psychiatry 15: 185-203, 2010.

14. Bu Q, Yang Y, Yan G, Hu Z, Hu C, Duan J, Lv L, Zhou J, Zhao J, Shao X, et al: Proteomic analysis of the nucleus accumbens in rhesus monkeys of morphine dependence and withdrawal intervention. J Proteomics 75: 1330-1342, 2012.

15. Shevchenko A, Wilm M, Vorm O and Mann M: Mass spectrometric sequencing of proteins silver-stained polyacrylamide gels. Anal Chem 68: 850-858, 1996. 
16. de Groot E, Hovingh GK, Wiegman A, Duriez P, Smit AJ, Fruchart JC and Kastelein JJ: Measurement of arterial wall thickness as a surrogate marker for atherosclerosis. Circulation 109 (23 Suppl 1): III33-III38, 2004.

17. Apple FS and Collinson PO; IFCC Task Force on Clinical Applications of Cardiac Biomarkers. Analytical characteristics of high-sensitivity cardiac troponin assays. Clin Chem 58: 54-61, 2012.

18. Di Angelantonio E, Chowdhury R, Sarwar N, Ray KK, Gobin R, Saleheen D, Thompson A, Gudnason V, Sattar N and Danesh J: B-type natriuretic peptides and cardiovascular risk: Systematic review and meta-analysis of 40 prospective studies. Circulation 120: 2177-2187, 2009.

19. Abraham JD,Calvayrac-Pawlowski S, Cobo S, Salvetat N, Vicat G, Molina L, Touchon J, Michel BF, Molina F, Verdier JM, et al: Combined measurement of PEDF, haptoglobin and tau in cerebrospinal fluid improves the diagnostic discrimination between alzheimer's disease and other dementias. Biomarkers 16: 161-171, 2011.

20. Rashedinia M, Lari P, Abnous K and Hosseinzadeh H: Proteomic analysis of rat cerebral cortex following subchronic acrolein toxicity. Toxicol Appl Pharmacol 272: 199-207, 2013.

21. Zgave T, Hu TT, Van de Plas B, Vinken M, Ceulemans AG, Hachimi-Idrissi S, Sarre S, Michotte Y and Arckens L: Proteomic analysis of global protein expression changes in the endothelin-1 rat model for cerebral ischemia: Rescue effect of mild hypothermia. Neurochem Int 63: 379-388, 2013.

22. Lu W, Wan X, Liu B, Rong X, Zhu L, Li P, Li J, Wang L, Cui L and Wang X: Specific changes of serum proteins in Parkinson's disease patients. PLoS One 9: e95684, 2014.

23. Alberio T, Bucci EM, Natale M, Bonino D, Di Giovanni M, Bottacchi E and Fasano M: Parkinson's disease plasma biomarkers: An automated literature analysis followed by experimental validation. J Proteomics 90: 107-114, 2013.
24. Arnoldi R, Hiltbrunner A, Dugina V, Tille JC and Chaponnier C: Smooth muscle actin isoforms: A tug of war between contraction and compliance. Eur J Cell Biol 92: 187-200, 2013.

25. Papke CL, Cao J, Kwartler CS, Villamizar C, Byanova KL, Lim SM, Sreenivasappa H, Fischer G, Pham J, Rees M, et al: Smooth muscle hyperplasia due to loss of smooth muscle $\alpha$-actin is driven by activation of focal adhesion kinase, altered p53 localization and increased levels of platelet-derived growth factor receptor- $\beta$. Hum Mol Genet 22: 3123-3137, 2013.

26. Kern S, Feng HZ, Wei H, Cala S and Jin JP: Up-regulation of alpha-smooth muscle actin in cardiomyocytes from non-hypertrophic and non-failing transgenic mouse hearts expressing $\mathrm{N}$-terminal truncated cardiac troponin I. FEBS Open Bio 4: 11-17, 2013.

27. Simoneau B, Houle F and Huot J: Regulation of endothelial permeability and transendothelial migration of cancer cells by tropomyosin-1 phosphorylation. Vasc Cell 4: 18, 2012.

28. Wieten L, Berlo SE, Ten Brink CB, van Kooten PJ, Singh M, van der Zee R, Glant TT, Broere F and van Eden W: IL-10 is critically involved in mycobacterial HSP70 induced suppression of proteoglycan-induced arthritis. PLoS One 4: e4186, 2009.

29. Tirapelli DP, Carlotti Jr CG, Leite JP, Tirapelli LF and Colli BO: Expression of HSP70 in cerebral ischemia and neuroprotetive action of hypothermia and ketoprofen. Arq Neuropsiquiatr 68: 592-596, 2010.

30. Verma P, Pfister JA, Mallick S and D'Mello SR: HSF1 protects neurons through a novel trimerization- and HSP-independent mechanism. J Neurosci 34: 1599-1612, 2014. 